\title{
Molecular clocks without rocks: new solutions for old problems
}

\section{2}

George P. Tiley ${ }^{1}$, Jelmer W. Poelstra ${ }^{1}$, Mario dos Reis ${ }^{2}$, Ziheng Yang ${ }^{3}$, Anne D. Yoder ${ }^{1,{ }^{*}}$ ${ }^{1}$ Department of Biology, Duke University, Durham, NC 27708, USA

${ }^{2}$ School of Biological and Chemical Sciences, Queen Mary University of London, London E1 4NS, UK

${ }^{3}$ Department of Genetics, Evolution, and Environment, University College London, London WC1E 6BT, UK

*Correspondence: anne.yoder@duke.edu

Keywords: Divergence Time Estimation, Multispecies Coalescent, Substitution Rate, Mutation Rate, Effective Population Size, Gene Tree Discordance

\section{Abstract}

Molecular data have been used to date species divergences ever since they were described as "documents of evolutionary history" in the 1960s. Yet, an inadequate fossil record and

discordance between gene trees and species trees are persistently problematic. We examine how, by accommodating gene tree discordance and by scaling branch lengths to absolute time using mutation rate and generation time, multispecies coalescent (MSC) methods can potentially overcome these challenges. We find that time estimates can differ - in some cases,

1 substantially - depending on whether MSC methods or traditional phylogenetic methods that apply concatenation are used, and whether the tree is calibrated with pedigree-based mutation rates or with fossils. We discuss the advantages and shortcomings of both approaches and provide practical guidance for data analysis when using these methods. 


\section{Divergence Time Estimation}

28 Zukerkandl and Pauling [1] were the first to posit that genetic distances between organisms

29 could be converted to absolute geological times, describing genomes as "documents of

30 evolutionary history." The most commonly used molecular clock methods (See Glossary)

31 estimate absolute times from genetic distances by calibrating the species tree with fossil data,

32 assuming either a constant rate of evolution among lineages (the molecular clock) or variable

33 rates (relaxed clock models) [2-4]). Recently, the multispecies coalescent (MSC) [5] is on

34 the ascent as a method for estimating divergence times [6, 7] due at least in part to potential

35 freedom from fossil calibrations [8, 9]. However, conflicts can arise in empirical studies

36 between traditional phylogenetic clock models and MSC methods, raising the question of

37 which method is more reliable for placing evolutionary events in a temporal context. Here, we

38 examine the fundamental assumptions and analytical details of these two general

39 methodological classes: 1) Traditional phylogenetic clock models that use concatenation of

40 genetic loci and 2) MSC models that explicitly model gene tree discordance due to

41 incomplete lineage sorting (ILS). Both approaches can be used without fossil calibrations

42 where a priori information on absolute rates of evolution are available, but some features of the

43 MSC are ideal for estimating species divergence times by leveraging external de novo

44 mutation rate $(\mu)$ estimates, typically measured from pedigree trios (Fig. 1; Key Figure). We

45 conclude by describing conditions that influence the suitability of the two approaches and offer

46 recommendations for the proper application of both.

48 The Allure of the Molecular Clock

49 Clock models and their applications have had enormous impacts on our understanding of the

50 history of life on earth, including the timing of life history transitions [10], global ecological

51 change in response to climate oscillations [11], the ancient origins of orders such as

52 Lepidoptera [12], and even the origin of life or the last universal common ancestor (LUCA) after 
53 the Moon-forming impact [13]. Calibration of the molecular clock has historically been

54 performed using fossil ages [14] or geological events [15] though only a tiny fraction of

55 phylogenetic lineages have reliable fossil records for appropriate calibration [16]. Thus, the lack

56 of a detailed fossil record for many groups is a major constraint for investigating the evolutionary

57 history of those lineages. For instance, both plant [17] and animal fossils [18] are difficult to

58 characterize in tropical rainforests where available rock formations for fossilization are typically

59 absent [19]. In some groups, such as grasses, calibrations based on phytolith microfossils are

60 contentious because of ambiguous diagnostic characters that compromise accurate

61 phylogenetic placement [20]. And for many groups, such as the glass frogs [21], fossils are

62 entirely absent.

64 Thus, for the analysis of most clades across the tree of life, investigators must depend on fossil

65 calibrations that are phylogenetically distant from the organisms of interest. As phylogenetic

66 distance increases, the complexities of modeling rate variation among lineages also increases

67 given the now extensive evidence that molecular rates change frequently across phylogeny.

68 Finally, a growing body of literature suggests that by ignoring genetic polymorphism in ancestral

69 species, divergence times may be systematically biased [6, 7, 9].

\section{Relaxed Clock Models}

72 When a calibration point can be placed with confidence within a given clade and close to its

73 most recent common ancestor (MRCA), it is possible to estimate per-year substitution

74 rates. Using that rate, an investigator can then infer divergence times for other nodes in the

75 phylogeny that do not have fossil calibrations. This assumes, however, that all lineages share a

76 single rate of evolution: i.e., that there is a strict molecular clock. While this is not an

77 unreasonable assumption for closely related species, the strict clock is typically violated when

78 more distantly related species are included [22]. Such violations can arise not only from 
differences in the molecular mechanisms that generate mutations [23], but also from variation in

80 life history traits [24, 25]. For example, great apes have lower substitution rates compared to

81 Old World and New World monkeys (the hominoid slowdown hypothesis [26]), a phenomenon

82 that can largely be explained by differences in generation time among species [27]. Similar

83 observations have been made in plants by comparing woody and herbaceous species [28, 29].

85 The clock can be "relaxed" by allowing for variable rates among branches on a phylogeny while

86 maintaining computational tractability and statistical identifiability [2, 3, 30, 31]. The first relaxed

87 clock methods that could leverage uncertainty across multiple calibrations were implemented

88 with maximum likelihood and required a priori assumptions to partition branches into different

89 rate groups (e.g., "local clocks" [32] or heuristic approaches [33, 34]). Recent Bayesian

90 methods have incorporated uncertainty in calibrations and as well as in rates of evolution

91 through the use of prior distributions. Different models of rate variation among branches are

92 available, including autocorrelation among lineages [2, 35], uncorrelated rates [3, 30, 36], or a

93 mixture of the two [37]. However, per-year substitution rates and divergence times are sensitive

94 to prior distribution on node calibrations [38] and justifying informed node calibrations is not

95 trivial [39]. Relaxed clock methods have recently been extended to account for uncertainty in

96 fossil placement [40] by leveraging morphological data from both extant and fossil species [41-

97 44]. These total-evidence [41] approaches include tip-dating methods that treat extinct fossil

98 lineages as tips where fossil occurrence [40] or morphological characters from fossils [43] can

99 calibrate rates of evolution to absolute time. They can also incorporate different speciation

100 mechanisms that best suit an organismal group [45]. As with more traditional methods,

101 however, these total-evidence tip-dating methods can only be applied to clades with an

102 available fossil record [42] and therefore cannot solve the problem of poor or absent fossil

103 records.

104 
105 Tip-dating methods that use only molecular data [46, 47] offer one approach for overcoming an

106 absence of fossil calibrations. These methods have been applied to viruses, where high

107 substitution rates generate sufficient variation from contemporary samples to determine relative

108 ages [48], as well as to cases wherein ancient DNA samples can calibrate the molecular clock

109 such as for woolly mammoths [49] and humans [50]. Even so, ancient DNA methods are

110 equally or even more restrictive than fossil-calibrated methods given that they can only be

111 applied to a limited number of organisms for which well-preserved and relatively recent samples

112 are available [51]. Most significantly, all of the above methods use concatenation of genetic

113 loci, thereby making the fundamental assumption that the phylogenetic history of each locus

114 matches the species tree. We here discuss how concatenation can be problematic, and how

115 MSC methods overcome these problems.

117 The Multispecies Coalescent as a Backward Time Machine

118 Coalescent theory is a branch of population genetics that describes the genealogical histories

119 of a sample of alleles in a population, going back from a sample of extant alleles to their most

120 recent common ancestor [52]. Two alleles are said to coalesce when they share a common

121 ancestor. The MSC is a simple extension of the single-population coalescent to multiple species

122 [5] and accommodates the species phylogeny and the coalescent processes in both the extant

123 and extinct species $[53,54]$. The MSC jointly estimates divergence times and rates of

124 evolution (Fig. 1) while explicitly modeling gene tree discordance due to incomplete lineage

125 sorting (ILS, also known as deep coalescence). ILS occurs when sequences from different

126 species fail to coalesce in their most recent ancestral species. The shorter the branch in

127 coalescent units between two speciation events, the more likely is ILS to occur (Box 1). Short

128 coalescent branch lengths can be caused not only by small time intervals between speciation

129 events, but also by large ancestral effective population size. 
131 It is now well accepted that gene trees do not consistently match species trees [55]. Though this

132 was initially considered to be a hindrance to the accurate reconstruction of phylogenies [56],

133 investigators are increasingly aware that these heterogeneities provide valuable information

134 about the timing and population dynamics of organismal lineages over their evolutionary history.

135 Described as a "backward time machine" [57], the MSC treats the stochastic variation of the

136 coalescent process over genes or genomic regions as a source of information rather than as

137 "mistakes" or "conflicts", and is thus uniquely suited to harness the power of many loci from

138 modern genomic data. Accordingly, the MSC is of increasing interest to investigators who seek

139 to place divergence events in a temporal context. The MSC makes a number of simplifying

140 assumptions including a lack of post-divergence gene flow, ILS as the only source of gene tree

141 discordance, no recombination within loci, and a lack of selection. Where high amounts of gene

142 flow among non-sister species are a concern, extensions to the MSC are available [58].

\section{Accounting for the Coalescent Process}

145 Traditional phylogenetic clock models equate species divergence (i.e., "split times") to sequence

146 divergence. This is problematic given that sequence divergence will always predate speciation

147 events in the absence of gene flow [59, 60] (Fig. 2). In contrast, coalescent methods explicitly

148 accommodate the differences between the two and directly estimate species divergence times

149 which are generally the evolutionary events of interest (Fig. 3; Box 2). Moreover, when fossil

150 calibrations are used, divergence time estimates can be strongly affected, with the direction of

151 the bias depending on the placement of the most precise calibrations. If these calibrations are

152 placed on young nodes within a phylogeny, divergence times will be underestimated across the

153 entire phylogeny, while if calibrations are placed on ancient nodes, the ages of young nodes are

154 likely to be overestimated. Accordingly, for phylogenies with complex mixtures of fossil

155 calibrations, both underestimation and overestimation of divergence times may occur across the 156 phylogeny - regardless of the analytic method applied. 
158 Traditional phylogenetic analysis of concatenated sequences assumes that a single tree 159 topology with one set of divergence times underlies the multilocus sequence data, irrespective 160 of how rate variation is modeled among sites, loci, or branches. Gene tree discordance due to

161 ILS then appears as additional substitutions on branches in the species phylogeny [6, 61],

162 leading to overestimation of species divergence times when ILS is not accounted for [9]. In line

163 with these theoretical expectations, Stange et al. [7] showed that in cases of high gene tree

164 discordance, concatenation methods will overestimate ages of young nodes when ancient

165 nodes are constrained. Similarly, Fang et al. [62] found that recent species divergences were

166 correctly estimated to be more recent when using MSC methods. Simulations generally suggest

167 that the MSC can improve divergence time estimates when gene tree discordance is high [7, 9],

168 while comparable performance should be expected between concatenation and MSC methods

169 when gene tree discordance is low (Fig. 3).

171 Although empirical studies using MSC approaches have thus far focused on recent species

172 divergences (1-10 MYA) [7, 62, 63], the effects of discordant gene trees should also impact

173 divergence time estimates for older divergences where the coalescent branch length is short

174 and ILS is high [9]. These patterns are expected for rapid radiations that occurred deep in

175 evolutionary history, such as placental mammals [64], passerine birds [65], and lepidopterans

176 [12]. Divergence time estimates for these groups are important for interpreting species

177 biogeography and trait evolution, and as computational efficiency and resources continue to

178 improve, the evolutionary history of these groups should be re-evaluated with MSC models that

179 also leverage fossil calibrations. In angiosperms, reconciliation of molecular dates with those

180 interpreted from the fossil record has been the topic of vigorous debate even though molecular

181 data have largely been restricted to chloroplast sequences, which represent a single gene tree 
182 [66-69]. As large multilocus nuclear datasets become increasingly available for plants [70], the 183 benefits of fossil-calibrated MSC methods could be realized.

\section{The Coalescent Time Unit}

186 Because the average coalescence time between two randomly sampled sequences from a 187 diploid population is $2 \mathrm{~N}$ generations, it is convenient to scale branch lengths in the species tree 188 in coalescent units, that is, to use $T=t /(2 N)$ where $t$ is the number of generations until the 189 coalescent event. $T$ can also be rescaled by mutations and represented as $\tau=\mu t$, where $\mu$ is the 190 per-generation mutation rate, so that $T=\tau(\theta / 2)$. Here $\theta=4 N \mu$ is the population-scaled

191 mutation rate; a fundamental parameter in population genetic models which represents the 192 average number of mutations per site between two sequences randomly sampled from the 193 population.

195 MSC programs like StarBEAST2 [6] and BPP $[5,71]$ use multilocus sequence alignments to 196 estimate species trees as well as parameters in the MSC model including species divergence 197 times $(\tau)$ and population sizes (BPP estimates $\theta$ and StarBEAST2 estimates $N \mu$ ), both 198 measured by the expected number of mutations per site. If fossil calibrations or mutation rates 199 are available to calibrate the tree, they can be used to convert genetic distance to absolute 200 times and absolute rates. When a per-generation mutation rate is available, generation times

201 are also necessary (Fig. 1) to convert to divergence times in years. This approach assumes that 202 the per-generation mutation rate and generation time are constant throughout the species tree, 203 which is a reasonable assumption for analyses of closely related species for which genetic 204 divergences likely satisfy a strict clock [72, 73]. 
207 In order to estimate absolute divergence times in the absence of fossil calibrations, direct

208 estimates of the mutation rate estimates are needed. Recently, whole-genome sequencing data

209 from pedigree trios have been used to estimate the de novo mutation rate for many animals

210 [74-78] and parent-progeny pairs in plants [79]. Recent examples of divergence time estimation

211 based on mutation rates and coalescent age estimates include the age of human migration

212 events [80] and of domestication histories among agriculturally important species [81-83].

213

214 To estimate a de novo mutation rate, the father, mother, and offspring from a pedigree trio are

215 sequenced and aligned to a reference genome. Variants detected in the child that are distinct

216 from both the mother and father and do not match the reference are considered de novo

217 mutations. Because the number of sequencing errors are more than an order of magnitude

218 greater than the number of true mutations, strict filtering criteria in computational analysis must

219 be applied to the called variants to avoid false positives. Also, mutations cannot be identified at

220 all sites because of variable sequencing read depth and alignment uncertainty in repetitive

221 regions. Thus, the number of callable sites needs to be estimated as the denominator to

222 accurately estimate $\mu$ [76]. Ideally, the final estimate of $\mu$ is averaged over multiple pedigrees,

223 as any single pedigree will yield few mutations. Best practices for reducing false positives and

224 false negatives for inferred mutations are still being developed [84].

226 The availability of a reference genome can be a critical limitation for estimating de novo

227 mutation rates in non-model organisms. Although high-quality reference genomes are

228 anticipated for most Eukaryotic lineages in the near future [85], there will ultimately be barriers

229 for some groups. In the absence of direct estimates of $\mu$ for a species of interest, distributions of

$230 \mu$ can be developed based on studies of related organisms [72]. Generation time estimates must

231 be considered as well given that mutation rates from pedigree studies are scaled by generation

232 to recover absolute divergence times (Fig. 1). 
234 Discrepancies between Concatenation and MSC methods for Divergence Time Estimates

235 Though empirical examples are as yet few, discrepancies between divergence dates estimated 236 by fossil-calibrated concatenation and mutation rate-calibrated MSC methods are emerging

237 (e.g., Fig 4). For the closely related species pair of human and chimpanzees, the mutation rate-

238 calibrated MSC [9] and concatenated time estimation give similar results. Fossil-calibrated

239 concatenation and fossil-calibrated MSC methods place the divergence between 5.7 and 10

240 MYA, typically near the center of the calibration density at 7.5 MYA $[9,86]$. A mutation rate-

241 calibrated MSC analysis that assumed the human mutation rate for both species recovered a

242 posterior mean of 8.2 MYA [9]. Divergence time estimates calibrated directly with mutation rates

243 but not using the MSC are also similar, but only after considering the difference between

244 species and sequence divergence. In one such study, pairwise sequence divergence

245 between chimp and human ( $t_{\text {Seq; }}$ Fig. 2 ) yielded a divergence time of 12.1 MYA assuming the

246 human mutation rate [27], though subtracting $2 N_{H C}$ (the effective population size for the human-

247 chimpanzee common ancestor) yields a divergence time of 7.9 MYA. Thus, per-generation

248 mutation rates can be used to estimate divergence times from concatenated data too, but the

249 difference between species divergence and sequence divergence needs to be accommodated

250 by some calculation of population size estimate (Fig. 2).

252 The sensitivity of these methods to the mutation rate estimate is keen. For example, when the 253 human mutation rate was applied unilaterally across a primate phylogeny, a divergence time 254 between Old world monkeys (Macaca mulatta) and humans of 62 MYA was recovered [87], in 255 stark contrast to the 35 MYA age estimate indicated by fossil evidence [86]. The discrepancy is 256 likely explained by a slower mutation rate in humans compared to Old World monkeys [88] and

257 indicates that caution is needed when applying pedigree-based mutation rates to divergence 258 time estimation, especially across large phylogenies. While one possible reason for 
discrepancies across long times scales is that purifying selection may lead to lower substitution

260 rates compared to mutation rates [89], as observed in mutation accumulation lines with

261 Arabidopsis thaliana [90], the discrepancy in this case was in the opposite direction. Thus, given

262 the small number of empirical examples at present, it is difficult to generalize the causes of

263 disparities between substitution and mutation rates at present.

265 In one such empirical example, MSC methods produce significantly more recent age estimates 266 than fossil-calibrated concatenation methods for mouse lemurs (genus Microcebus). Whereas a 267 mutation rate-calibrated MSC analysis yields a MRCA for the genus of 1.5 MYA [63], previous 268 analyses using fossil-calibrated concatenation methods yielded estimates of $\sim 10$ MYA [86, 91].

269 Though this discrepancy could, in part, be the consequence of a falsely elevated pedigree-

270 based mutation rate estimate [91], the discrepancy would still be pronounced even if the true 271 rate is only half of the estimated rate. Conversely, for the fossil-calibrated divergence time 272 estimate using concatenation, phylogenetically distant, external calibrations [86, 91] were used 273 by necessity given that there is a complete dearth of fossils within the lemuriform clade. As 274 described above, the fossil-calibrated concatenation estimate is thus likely to overestimate 275 divergence times for young nodes given the dependence on older fossil calibrations deeper in 276 the phylogeny (Fig. 3; [9]). This is similar to cases of Stange et al. [7] and Fang et al. [62] where 277 MSC methods using geological calibrations resulted in more recent divergence times compared 278 with those found with concatenation - even when using the same calibrations. In summary, it is 279 important to note that the differences in time estimates between the MSC and phylogenetic 280 concatenation methods may be complex, depending on biases of mutation rate estimates and 281 on the relative placement of calibrations within the phylogeny. 
284 Divergence time estimates can fundamentally affect interpretations of trait evolution,

285 biogeography, and the processes that underlie species radiations. Thus, the stakes for

286 evolutionary studies are high. As an important step forward, future studies that leverage

287 genomic data and fossil calibrations should consider comparing traditional phylogenetic clock

288 models and the MSC to evaluate the effects of ILS on divergence time estimation. We further

289 recommend that uncertainty in both mutation rates and generation times should be explicitly

290 incorporated in analyses wherein coalescent units are converted to absolute time [63, 72]. This

291 can be easily done by drawing mutation rates and generation times from prior distributions

292 rather than relying on point estimates, given that variation in inferred mutation rates can be high

293 among pedigrees [84], and mutation rates may change over time [27]. Moreover, estimating

294 generation times can be problematic, especially for perennial plants given the lack of clear

295 segregation in the germ line. The impact of the number, quality, and placement of fossil

296 calibrations - as well as model choice on divergence time estimation using traditional

297 phylogenetic concatenation methods - has been extensively studied [10, 38, 67, 69, 86].

298 Conversely, the careful evaluation of MSC methods for divergence time estimation is still in its

299 infancy. We therefore predict that future studies that directly compare the two approaches are

300 likely to identify as yet unrecognized though critical considerations for accurate divergence time 301 analysis.

\section{Concluding Remarks}

304 We conclude by noting that despite its advantages, the MSC method involves a heavy

305 computational burden and may not always be feasible for divergence time estimation on large

306 phylogenies [92-94]. In such cases, traditional phylogenetic clock analyses that use

307 concatenation may be the most practical approach [3, 30]. In particular, approximate likelihood

308 calculation appears useful in estimating divergence times for large phylogenies or for very long

309 alignments [86]. These models should not be seriously biased when divergence times are old 
310 (Fig. 2) and ILS is low (Fig. 3). But given the prevalence of ILS across the tree of life, the

311 applications of the MSC for divergence time estimation in both shallow and deep phylogenies

312 will be of increasing interest and importance (see Outstanding Questions). It remains to be

313 seen to what degree divergence time estimates will agree when both traditional phylogenetic

314 clock models and mutation-rate calibrated MSC methods are applied within the same study

315 systems.

316

\section{Acknowledgements}

318 We thank Priya Moorjani for her helpful comments on an early version. The editor and two

319 anonymous reviewers provided comments that improved the manuscript. ADY gratefully

320 acknowledges the John Simon Guggenheim Foundation and the Alexander von Humboldt

321 Foundation for their support. ZY is supported by a Biotechnology and Biological Sciences

322 Research Council grant (BB/P006493/1). 


\section{Glossary}

324 Ancient DNA Methods: Sequence data is obtained from the remains of ancient specimens.

325 The DNA is typically damaged and fragmented by absolute time and by exposure to damaging 326 agents such as heat, oxidation, and UV irradiation.

327 Bayesian Methods: Bayes theorem is used to approximate the maximum likelihood estimates

328 of a model and its parameters by sampling many estimates with proposal distributions and

329 commonly implemented with a Markov chain Monte Carlo algorithm. Prior distributions are used

330 to constrain the search space of parameters and may include a priori expectations for

331 parameter estimates but are often left vague. Bayesian methods are used as a matter of

332 computational convenience when maximum likelihood optimization is intractable.

333 Callable Sites: The number of sites where a de novo mutation should be detectable.

334 Concatenation: Multiple loci are treated as a single nonrecombining locus with a single

335 underlying topology.

336 Coalescent: The stochastic process of lineage joining when one traces the genealogical history

337 of a sample of sequences from a population backwards in time.

338 Coalescent Age Estimate: The divergence time for two sequences based on sampling theory

339 and measured in the expected number of generations.

340 Coalescent Time Unit: The expected coalescent time for a pair of sequences, which is $2 \mathrm{~N}$

341 generations for a diploid species with population size $N$.

342 de novo Mutation Rate: The spontaneous germline mutation rate revealed in comparisons of

343 whole genomes from both parents and their progeny (aka, pedigree trios).

344 Deep Phylogenies: Phylogenies that contain species with high sequence divergence. In such

345 cases, substitutional saturation or "multiple hits," long-branch attraction, gene duplication and

346 loss, and model misspecification can result in gene tree discordance. ILS can still be a

347 substantial source of conflict between gene trees and species trees in deep phylogenies. 
348 Divergence Times: The expected absolute age at which two species became isolated from

349 each other.

350 Effective Population Size: The number of individuals that would produce the observed rate of 351 genetic drift in an idealized Fisher-Wright population model.

352 Fossil Calibrations: Fossil evidence from morphological characters that can constrain the age

353 of the crown group of a clade (e.g., with a hard minimum and a soft maximum).

354 Gene Flow: Exchange of alleles between two populations.

355 Gene Tree: The evolutionary history of a short, nonrecombining segment of the genome

356 Gene Tree Discordance: Difference in gene tree topology from the species tree, possibly

357 caused by deep coalescence.

358 Generation Time: The average time between two generations which is often quantified as the

359 average age of parents at birth, averaged over individuals.

360 Lineage sorting: The process by which gene lineages become fixed within a species

361 such that all alleles within that species coalesce to a single ancestral allele within the

362 species lineage.

363 Incomplete Lineage Sorting (ILS): Failure for two sequences from two species to coalesce in

364 the most recent common ancestral species, also known as deep coalescence.

365 Loci: Orthologous non-recombining sequences. Each locus corresponds to an independent 366 gene tree.

367 Markov chain Monte Carlo (MCMC): a simulation approach for sampling from a target

368 distribution such as the posterior distribution of parameters in Bayesian inference.

369 Molecular Clock: Hypothesis that the rate of molecular evolution is constant over time and

370 among lineages.

371 Most Recent Common Ancestor (MRCA): The most recent node on a phylogeny from which 372 all individuals in a clade of interest are derived. 
373 Multispecies Coalescent (MSC): The extension of the coalescent process to multiple species

374 which accommodates the species tree as well as the coalescent within populations.

375 Pairwise Sequence Divergence: The evolutionary distance between a pair of sequences

376 measured as the expected number of substitutions per site.

377 Pedigree Trio: A child and the two parents for whom whole genomes are sequenced and

378 compared to identify the new mutations in the child.

379 Per-year Substitution Rates: The number of substitutions per-site per-year that are obtained

380 when calibrating a phylogeny to absolute time with information at nodes or tips.

381 Reciprocal Monophyly: All alleles within a species coalesce with each other before the first

382 coalescence with an allele from another species.

383 Relaxed Clock Models: An extension of the strict clock model to allow changes in evolutionary

384 rate over branches in a phylogeny.

385 Species Tree: The evolutionary history of species, which is often estimated from many

386 individual gene trees or loci.

387 Strict Molecular Clock: A single rate of molecular evolution is enforced for all branches in a 388 phylogeny.

389 Tip-Dating Methods: Rates of molecular evolution are calibrated to absolute time by known 390 sampling dates of individuals, whether extant or extinct, at the tips of a phylogeny.

391 Total-Evidence: Morphological characters for extinct (fossil) and extant tips and rates of

392 morphological evolution are used to infer species divergence times jointly with molecular data.

393 Traditional Phylogenetic Clock Models: Models for divergence time estimation that assume 394 one tree and one set of divergence times for all loci. 
Box Legends

397 Box 1 - Incomplete Lineage Sorting on a Rooted Three-Taxon Species Tree.

398 Box 2 - Differences between Bayesian methods for divergence time estimation and 399 programs for implementing them.

$401 \quad$ Figure Legends

402 Figure 1 - Overview of the MSC model and its use for estimating absolute divergence 403 times with external mutation rate data. Input Data - The MSC requires aligned orthologous 404 sequence data as input. There can be many individual loci, and each locus is assumed to be 405 non-recombining and not under selection. The MSC allows for multiple alleles per species per 406 locus, and sampling multiple alleles can improve parameter estimates. Although joint estimation 407 of the species tree and MSC parameters is possible, using a fixed species tree is 408 computationally more efficient. Estimate MSC Parameters by MCMC - The MSC estimates 409 model parameters with Bayesian Markov chain Monte Carlo (MCMC). This requires a prior

410 distribution for all model parameters including population sizes $(\theta)$, species divergence times $(\tau)$, 411 and possibly rates among loci $(r)$. The MSC estimates a gene tree for each locus $(1 . . n)$ and the 412 coalescent times on those trees. Gene tees can be incongruent with the species tree. The 413 distribution of gene trees and their coalescent age estimates are used to jointly estimate $\theta$ and $\tau$ 414 on the species tree. Calibrate with Mutation Rate - A per-generation mutation rate $(\mu)$ is 415 obtained from independent pedigree-based studies and a generation time $(g)$ is estimated from 416 the distribution of parent ages at the time of birth for offspring. $\mu$ and $g$ can then be used to 417 obtain an absolute rate of evolution by multiplying $\tau$ by $g / \mu$. $\mu$ can also be used to rearrange the 418 expression for $\theta$ and obtain absolute population sizes $(N)$. 
420 Figure 2 - Overestimation of species divergence times from genetic distances. a) Species

$421 \mathrm{~A}$ and $\mathrm{B}$ diverged $t_{A B}$ generations ago. Sequences $\mathrm{A}$ and $\mathrm{B}$ coalesced further back in time at $t_{\text {Seq }}$

422 generations ago, with a mean $t_{S e q}-t_{A B}$ of $2 N_{A B}$ generations. b) Sequences were simulated under

423 the MSC for a pair of species with constant $N_{A B}=10^{5}$ and $\mu=1 \times 10^{-8}$ per site per generation. c)

424 The relative expected overestimation of species divergence times by $2 N_{A B}$ becomes smaller as

$425 t_{A B}$ becomes larger, because $2 N_{A B}$ contributes only a small proportion of time to the overall

426 divergence time estimate.

428 Figure 3 - Effects of ILS and the MSC on divergence time estimation. Data are from Table

4292 of Angelis and dos Reis [9]. a) Three-taxon species tree used for simulation. All data were

430 simulated under the MSC with the Jukes Cantor model of molecular evolution. A $\mu$ of $1 \times 10^{-8}$

431 per site per generation and a generation time of ten years was used, and the species tree root

432 (node $r$ ) had an age of 10 MYA or $\tau$ of 0.01 . Data were simulated with 4 different population

433 sizes $(N)$ that were constant along the species tree. The root was calibrated with a gamma

434 distribution as one might in a fossil-calibrated divergence time analysis. b) Divergence time

435 estimates of node s when using concatenation (MCMCTREE) or the MSC (BPP). Because the 436 calibration is placed on the older root node, the younger node is overestimated when $N$ or ILS is

437 high by concatenation but not MSC methods. Points represent posterior means and error bars 438 are the $95 \%$ credible intervals.

440 Figure 4 - Illustration of the consequences of differences between divergence time

441 estimates. Pedigree symbols represent mutation rate-calibrated divergence times and

442 probability distributions represent traditional phylogenetic clock model estimates. a) The MRCA

443 of Madagascar's mouse lemurs. The mutation rate-calibrated MSC estimate yields a mean

444 divergence time of 1.5 MYA whereas a traditional phylogenetic clock model with fossil 
445 calibrations recovers a divergence time estimate of approximately $10 \mathrm{Ma}$. Though the position of

446 Madagascar relative to Africa is essentially the same at these two geological time points,

447 Madagascar's climate would have been very similar to that of today at 1.5 MYA whereas it

448 would have been much warmer and drier 10 MYA. b) The divergence between Old World

449 monkeys and apes. A mutation rate-calibrated divergence time estimate (though not with the

$450 \mathrm{MSC}$ ) is 62 MYA whereas the traditional phylogenetic clock model yields a divergence time

451 estimate of approximately 35 MYA. There are striking differences in both continental

452 configuration and climate between these two time points. At 62 MYA, the earth was largely

453 tropical and sea levels were markedly high, isolating Africa from the northern continents. At 35

454 MYA, Africa has shifted northward, making contact with the northern continents and Antarctica

455 is partially glaciated indicating much cooler global temperatures. Global maps provided courtesy

456 of the Deep Time Maps project. 
1. Zuckerkandl, E. and Pauling, L. (1965) Molecules as documents of evolutionary history. J Theor Biol 8 (2), 357-66.

2. Thorne, J.L. et al. (1998) Estimating the rate of evolution of the rate of evolution. MBE 15 (12), 1647-1657.

3. Drummond, A.J. et al. (2006) Relaxed phylogenetics and dating with confidence. PLoS Biol 4 (5), e88.

4. dos Reis, M. et al. (2016) Bayesian molecular clock dating of species divergences in the genomics era. Nat Rev Genet 17 (2), 71-80.

5. Rannala, B. and Yang, Z. (2003) Bayes estimation of species divergence times and ancestral population sizes using DNA sequences from multiple loci. Genetics 164, 1645-1656.

6. Ogilvie, H.A. et al. (2017) StarBEAST2 Brings Faster Species Tree Inference and Accurate Estimates of Substitution Rates. Mol Biol Evol 34 (8), 2101-2114.

7. Stange, M. et al. (2018) Bayesian divergence-time estimation with genome-wide singlenucleotide polymorphism data of sea catfishes (Ariidae) supports Miocene closure of the Panamanian Isthmus. Syst Biol 67 (4), 681-699.

8. Burgess, R. and Yang, Z. (2008) Estimation of hominoid ancestral population sizes under bayesian coalescent models incorporating mutation rate variation and sequencing errors. Mol Biol Evol 25 (9), 1979-94.

9. Angelis, K. and dos Reis, M. (2015) The impact of ancestral population size and incomplete lineage sorting on Bayesian estimation of species divergence times. Current Zoology 61 (5), 874-885.

10. Liu, L. et al. (2017) Genomic evidence reveals a radiation of placental mammals uninterrupted by the KPg boundary. Proc Natl Acad Sci U S A 114 (35), E7282-E7290.

11. Hackel, J. et al. (2018) Grass diversification in Madagascar: In situ radiation of two large C3 shade clades and support for a Miocene to Pliocene origin of C4 grassy biomes. Journal of Biogeography 45 (4), 750-761.

12. Kawahara, A.Y. et al. (2019) Phylogenomics reveals the evolutionary timing and pattern of butterflies and moths. Proc Natl Acad Sci U S A 116 (45), 22657-22663.

13. Betts, H.C. et al. (2018) Integrated genomic and fossil evidence illuminates life's early evolution and eukaryote origin. Nat Ecol Evol 2 (10), 1556-1562.

14. Benton, M.J. and Donoghue, P.C. (2007) Paleontological evidence to date the tree of life. Mol Biol Evol 24 (1), 26-53.

15. De Baets, K. et al. (2016) Tectonic blocks and molecular clocks. Philos Trans R Soc Lond B Biol Sci 371 (1699).

16. Rannala, B. (2016) Conceptual issues in Bayesian divergence time estimation. Philos Trans R Soc Lond B Biol Sci 371 (1699). 

17. Wing, S.L. et al. (2009) Late Paleocene fossils from the Cerrejon Formation, Colombia, are the earliest record of Neotropical rainforest. Proc Natl Acad Sci U S A 106 (44), 18627-32.

18. Bloch, J.I. et al. (2016) First North American fossil monkey and early Miocene tropical biotic interchange. Nature 533 (7602), 243-6.

19. Kidwell, S.M. (2001) Major biases in the fossil record. In Paleobiology II (Briggs, D.E.G. and Crowther, P.R. eds), pp. 297-303, Blackwell Science Ltd.

20. Kergoat, G.J. et al. (2018) Opposite macroevolutionary responses to environmental changes in grasses and insects during the Neogene grassland expansion. Nat Commun 9 (1), 5089.

21. Castroviejo-Fisher, S. et al. (2014) Neotropical diversification seen through glassfrogs. Journal of Biogeography 41 (1), 66-80.

22. Langley, C.H. and Fitch, W.M. (1974) An examination of the constancy of the rate of molecular evolution. JME 3, 161-177.

23. Lynch, M. et al. (2016) Genetic drift, selection and the evolution of the mutation rate. Nat Rev Genet 17 (11), 704-714.

24. Li, W.-H. et al. (1996) Rates of nucleotide substitution in primates and rodents and the generation-time effect hypothesis. Molecular Phylogenetics and Evolution 5 (1), 182-187.

25. Mello, B. and Schrago, C.G. (2019) The estimated pacemaker for great apes supports the hominoid slowdown hypothesis. Evolutionary Bioinformatics 15, 1-8.

26. Goodman, M. (1985) Rates of molecular evolution: the hominoid slowdown. Bioessays 3 (1), 9-14.

27. Moorjani, P. et al. (2016) Variation in the molecular clock of primates. Proc Natl Acad Sci U S A 113 (38), 10607-12.

28. Smith, S.A. and Donoghue, M.J. (2008) Rates of molecular evolution are linked to life history in flowering plants. Science 322 (5898), 86-9.

29. Lanfear, R. et al. (2013) Taller plants have lower rates of molecular evolution. Nat Commun 4, 1879.

30. Rannala, B. and Yang, Z.H. (2007) Inferring speciation times under an episodic molecular clock. Systematic Biology 56 (3), 453-466.

31. Ho, S.Y. and Duchene, S. (2014) Molecular-clock methods for estimating evolutionary rates and timescales. Mol Ecol 23 (24), 5947-65.

32. Yoder, A.D. and Yang, Z.H. (2000) Estimation of primate speciation dates using local molecular clocks. MBE 17 (7), 1081-1090.

33. Sanderson, M.J. (1997) A nonparametric approach to estimating divergence times in the absence of rate constancy. MBE 14 (12), 1218-1231. 
34. Sanderson, M.J. (2002) Estimating absolute rates of molecular evolution and divergence times: a penalized likelihood approach. MBE 19 (1), 101-109.

35. Huelsenbeck, J.P. et al. (2000) A Compound Poisson Process for Relaxing the Molecular Clock. Genetics 154, 1879-1892.

36. Lepage, T. et al. (2007) A general comparison of relaxed molecular clock models. Mol Biol Evol 24 (12), 2669-80.

37. Lartillot, N. et al. (2016) A mixed relaxed clock model. Philos Trans R Soc Lond B Biol Sci 371 (1699).

38. Warnock, R.C. et al. (2015) Calibration uncertainty in molecular dating analyses: there is no substitute for the prior evaluation of time priors. Proc Biol Sci 282 (1798), 20141013.

39. Parham, J.F. et al. (2012) Best practices for justifying fossil calibrations. Syst Biol 61 (2), 346-59.

40. Heath, T.A. et al. (2014) The fossilized birth-death process for coherent calibration of divergence-time estimates. Proc Natl Acad Sci U S A 111 (29), E2957-66.

41. Ronquist, F. et al. (2012) A total-evidence approach to dating with fossils, applied to the early radiation of the hymenoptera. Syst Biol 61 (6), 973-99.

42. Zhang, C. et al. (2016) Total-Evidence Dating under the Fossilized Birth-Death Process. Syst Biol 65 (2), 228-49.

43. Gavryushkina, A. et al. (2017) Bayesian Total-Evidence Dating Reveals the Recent Crown Radiation of Penguins. Syst Biol 66 (1), 57-73.

44. Alvarez-Carretero, S. et al. (2019) Bayesian Estimation of Species Divergence Times Using Correlated Quantitative Characters. Syst Biol 68 (6), 967-986.

45. Silvestro, D. et al. (2018) Closing the gap between palaeontological and neontological speciation and extinction rate estimates. Nat Commun 9 (1), 5237.

46. Rambaut, A. (2000) Estimating the rate of molecular evolution: incorporating noncontemporaneous sequences into maximum likelihood phylogenies. Bioinformatics 16 (4), 395399.

47. Stadler, T. and Yang, Z.H. (2013) Dating phylogenies with sequentially sampled tips. Systematic Biology 62 (5), 674-688.

48. Worobey, M. et al. (2008) Direct evidence of extensive diversity of HIV-1 in Kinshasa by 1960. Nature 455 (7213), 661-4.

49. Chang, D. et al. (2017) The evolutionary and phylogeographic history of woolly mammoths: 608 
50. Llamas, B. and al., e. (2016) Ancient mitochondrial DNA provides high-resolution time scale of the peopling of the Americas. Sci. Adv.

51. Orlando, L. et al. (2013) Recalibrating Equus evolution using the genome sequence of an early Middle Pleistocene horse. Nature 499 (7456), 74-8.

52. Kingman, J.F.C. (1982) The coalescent. Stochastic Processes and their Applications 13, 235-248.

53. Xu, B. and Yang, Z. (2016) Challenges in Species Tree Estimation Under the Multispecies Coalescent Model. Genetics 204 (4), 1353-1368.

54. Rannala, B. et al. (2020) The multispecies coalescent model and species tree inference. In Phylogenetics in the Genomic Era (Scoenavacca, C. et al. eds), pp. 1-20.

55. Maddison, W.P. (1997) Gene Trees in Species Trees. Syst Biol 46 (3), 523-536.

56. Rokas, A. et al. (2003) Genome-scale approaches to resolving incongruence in molecular phylogenies. Nature 425, 798-804.

57. Yang, Z. (2014) Molecular Evolution: A Statisitical Approach, Oxford University Press.

58. Flouri, T. et al. (2020) A Bayesian Implementation of the Multispecies Coalescent Model with Introgression for Phylogenomic Analysis. Mol Biol Evol 37 (4), 1211-1223.

59. Edwards, S.V. and Beerli, P. (2000) Perspective: gene divergence, population divergence, and the variance in coalescence time in phylogeographic studies. Evolution 54 (6), 1839-1854.

60. Carstens, B.C. and Knowles, L.L. (2007) Estimating species phylogeny from gene-tree probabilities despite incomplete lineage sorting: An example from Melanoplus grasshoppers. Systematic Biology 56 (3), 400-411.

61. Mendes, F.K. and Hahn, M.W. (2016) Gene Tree Discordance Causes Apparent Substitution Rate Variation. Syst Biol 65 (4), 711-21.

62. Fang, B. et al. (2020) Estimating uncertainty in divergence times among three-spined stickleback clades using the multispecies coalescent. Mol Phylogenet Evol 142, 106646.

63. Schüßler, D. et al. (2019) Cryptic patterns of speciation in cryptic primates: microendemic mouse lemurs and the multispecies coalescent. bioRxiv.

64. Liu, W. et al. (2019) An evolutionarily stable strategy to colonize spatially extended habitats. Nature 575 (7784), 664-668.

65. Oliveros, C.H. et al. (2019) Earth history and the passerine superradiation. Proc Natl Acad Sci U S A 116 (16), 7916-7925.

66. Li, H.T. et al. (2019) Origin of angiosperms and the puzzle of the Jurassic gap. Nat Plants 5 (5), 461-470. 
67. Barba-Montoya, J. et al. (2018) Constraining uncertainty in the timescale of angiosperm evolution and the veracity of a Cretaceous Terrestrial Revolution. New Phytol 218 (2), 819-834.

68. Beaulieu, J.M. et al. (2015) Heterogeneous Rates of Molecular Evolution and Diversification Could Explain the Triassic Age Estimate for Angiosperms. Syst Biol 64 (5), 869-78.

69. Magallon, S. et al. (2015) A metacalibrated time-tree documents the early rise of flowering plant phylogenetic diversity. New Phytol 207 (2), 437-453.

70. Leebens-Mack and One Thousand Plant Transcriptomes, I. (2019) One thousand plant transcriptomes and the phylogenomics of green plants. Nature 574 (7780), 679-685.

71. Flouri, T. et al. (2018) Species Tree Inference with BPP Using Genomic Sequences and the Multispecies Coalescent. Mol Biol Evol 35 (10), 2585-2593.

72. Yoder, A.D. et al. (2016) Geogenetic patterns in mouse lemurs (genus Microcebus) reveal the ghosts of Madagascar's forests past. Proc Natl Acad Sci U S A 113 (29), 8049-56.

73. Federman, S. et al. (2018) Reconciling species diversity in a tropical plant clade (Canarium, Burseraceae). PLoS One 13 (6), e0198882.

74. Keightley, P.D. et al. (2015) Estimation of the spontaneous mutation rate in Heliconius melpomene. Mol Biol Evol 32 (1), 239-43.

75. Smeds, L. et al. (2016) Direct estimate of the rate of germline mutation in a bird. Genome Res 26 (9), 1211-8.

76. Pfeifer, S.P. (2017) Direct estimate of the spontaneous germ line mutation rate in African green monkeys. Evolution 71 (12), 2858-2870.

77. Thomas, G.W.C. et al. (2018) Reproductive longevity predicts mutation rates in primates. Curr Biol 28 (19), 3193-3197 e5.

78. Koch, E. et al. (2019) De novo mutation rate estimation in wolves of known pedigree. Mol Biol Evol.

79. Wang, L. et al. (2019) The architecture of intra-organism mutation rate variation in plants. PLoS Biol 17 (4), e3000191.

80. Pierron, D. et al. (2017) Genomic landscape of human diversity across Madagascar. Proc Natl Acad Sci U S A 114 (32), E6498-E6506.

81. Lawal, R.A. et al. (2020) The wild species genome ancestry of domestic chickens. BMC Biol $18(1), 13$.

82. Smith, O. et al. (2019) A domestication history of dynamic adaptation and genomic deterioration in Sorghum. Nat Plants 5 (4), 369-379.

83. Wu, D.D. et al. (2018) Pervasive introgression facilitated domestication and adaptation in the Bos species complex. Nat Ecol Evol 2 (7), 1139-1145. 
84. Smith, T.C.A. et al. (2018) Large scale variation in the rate of germ-line de novo mutation, base composition, divergence and diversity in humans. PLoS Genet 14 (3), e1007254.

85. Lewin, H.A. et al. (2018) Earth BioGenome Project: Sequencing life for the future of life. Proc Natl Acad Sci U S A 115 (17), 4325-4333.

86. dos Reis, M. et al. (2018) Using Phylogenomic Data to Explore the Effects of Relaxed Clocks and Calibration Strategies on Divergence Time Estimation: Primates as a Test Case. Syst Biol 67 (4), 594-615.

87. Moorjani, P. et al. (2016) Human Germline Mutation and the Erratic Evolutionary Clock. PLoS Biol 14 (10), e2000744.

88. Scally, A. et al. (2012) Insights into hominid evolution from the gorilla genome sequence. Nature 483 (7388), 169-75.

89. Ho, S.Y. et al. (2011) Bayesian estimation of substitution rates from ancient DNA sequences with low information content. Syst Biol 60 (3), 366-75.

90. Exposito-Alonso, M. et al. (2018) The rate and potential relevance of new mutations in a colonizing plant lineage. PLoS Genet 14 (2), e1007155.

91. Yang, Z.H. and Yoder, A.D. (2003) Comparison of likelihood and Bayesian methods for estimating divergence times using multiple gene loci and calibration points, with application to a radiation of cute-looking mouse lemur species. Systematic Biology 52 (5), 705-716.

92. Campbell, C.R. et al. (2019) Pedigree-based measurement of the de novo mutation rate in the gray mouse lemur reveals a high mutation rate, few mutations in $\mathrm{CpG}$ sites, and a weak sex bias. bioRxiv.

93. Zanne, A.E. et al. (2014) Three keys to the radiation of angiosperms into freezing environments. Nature 506 (7486), 89-92.

94. Jetz, W. et al. (2012) The global diversity of birds in space and time. Nature 491 (7424), 444-8.

95. Hudson, R.R. (1983) Testing the constant-rate neutral allele model with protein sequence data. Evolution 37 (1), 203-217.

96. Bouckaert, R. et al. (2019) BEAST 2.5: An advanced software platform for Bayesian evolutionary analysis. PLoS Computational Biology 15 (4), e1006650.

97. Yang, Z. (2007) PAML 4: phylogenetic analysis by maximum likelihood. Mol Biol Evol, 24 (8), 1586-1591.

98. Ronquist F. et al. (2012) MrBayes 3.2: Efficient Bayesian phylogenetic inference and model choice across a large model space. Systematic Biology 61 (3), 539-542.

99. Lartillot, N. and Philippe, H. A. (2004) A Bayesian mixture model for across-site heterogeneities in the amino acid replacement process. Mol Biol Evol, 21 (6), 1095-1109. 


\section{Highlights}

- Molecular clock models using fossil calibrations have allowed investigators to estimate the age of speciation events.

- Theoretical and computational developments have relaxed the assumption of a molecular clock, thus improving the accuracy of divergence time estimation.

- Despite these advances, estimates can be biased when there is widespread incomplete lineage sorting (ILS).

- Increased understanding of gene tree heterogeneity has driven multispecies coalescent (MSC) methods to prominence, though the potential power of the MSC for divergence time estimation remains largely unexplored.

- Absolute times can be obtained by using mutation rates estimated from pedigrees, providing [some] freedom from the incomplete fossil record.

- Mutation-rate calibrated MSC methods and traditional phylogenetic clock-dating methods with fossil calibrations can yield strikingly different divergence times. 


\section{Outstanding Questions}

- To what extent is among-lineage rate variation modeled by relaxed clock methods due to gene tree discordance from ILS?

- Have divergence times throughout the tree of life been systematically overestimated in clades that rely on external, and typically older, calibrations?

- Do divergence time estimates based on per-generation mutation rates and per-year substitution rates yield similar results, especially if substitution rates are estimated from presumably neutral regions of the genome such as third codon positions?

- Will MSC estimates that leverage fossil calibrations bring new insights to contentious age estimates such as the origins of placental mammals or angiosperms?

- Should effective population size variation among species be a concern for divergence time estimation studies using concatenation?

- Can mutation-rate calibrated MSC methods that account for variable rates and generation times among branches improve divergence time estimation for clades that have rapid life history transitions?

- How can we develop standard operating procedures for evaluating the strength of evidence for divergence time estimates from traditional phylogenetic analyses versus ages inferred from MSC methods that rely on mutation rate and generation time estimates?

- Are there alternative ways forward for estimating the absolute age of clades with poor or non-existent fossil representation?

- To what extent do the methods (MSC versus concatenation) and the calibrations (mutation rate versus fossils) impact divergence time estimates? 
Key IrnigutÐata

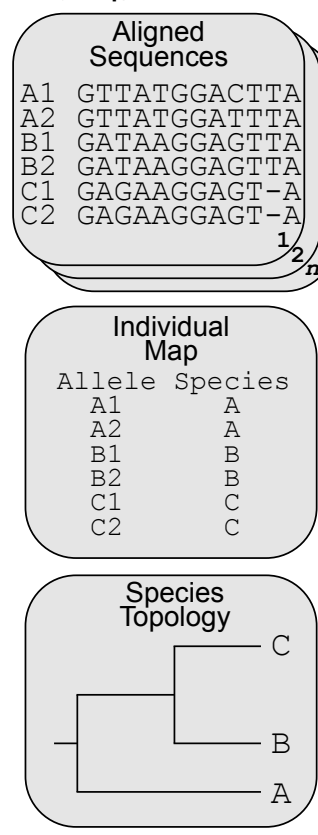

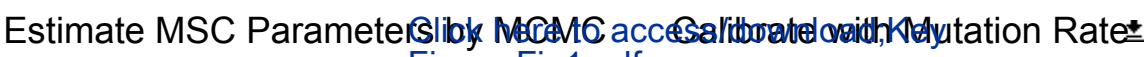
Figure;Fig1.pdf

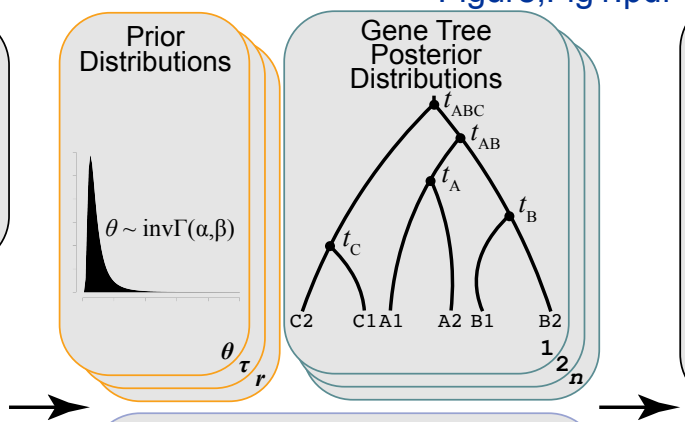

MSC Parameter Posterior Distributions

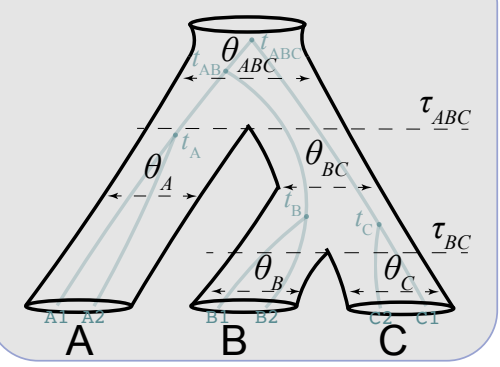

Mutation Rate Generation Time Estimation

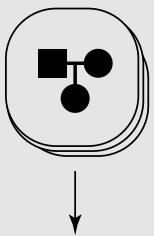

$\mu$

Absolute Divergence Time

$\tau=\mu t$ (MSC branch lengths)

$t$ (number of generations)

$\mu$ (mutations per site per generation)

$\mathrm{g}$ (number of years per generation)

Absolute Time $=\frac{\tau g}{\mu}=\operatorname{tg}$

Absolute Population Size

$\theta=4 N \mu$ (MSC ancestral population size)

Absolute Size $=\frac{\theta}{4 \mu}=N$
Estimation 


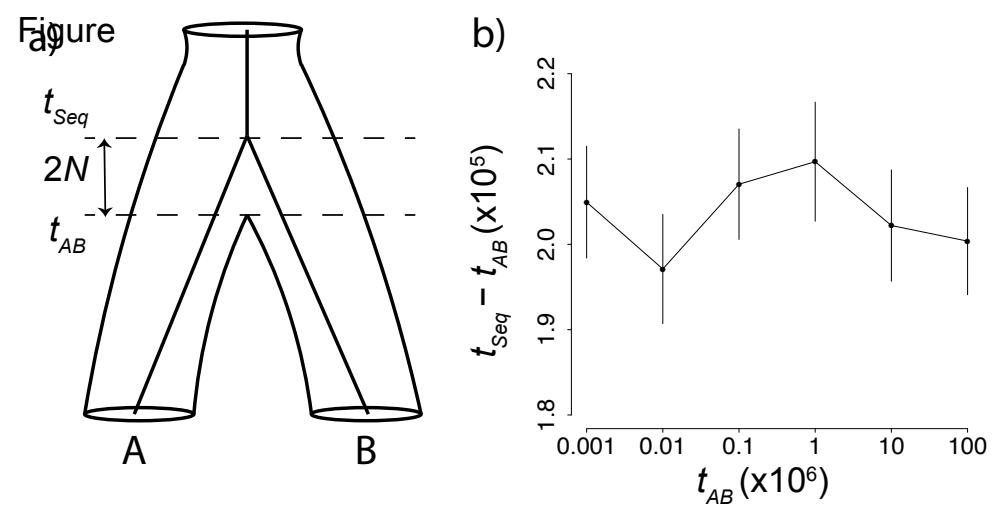



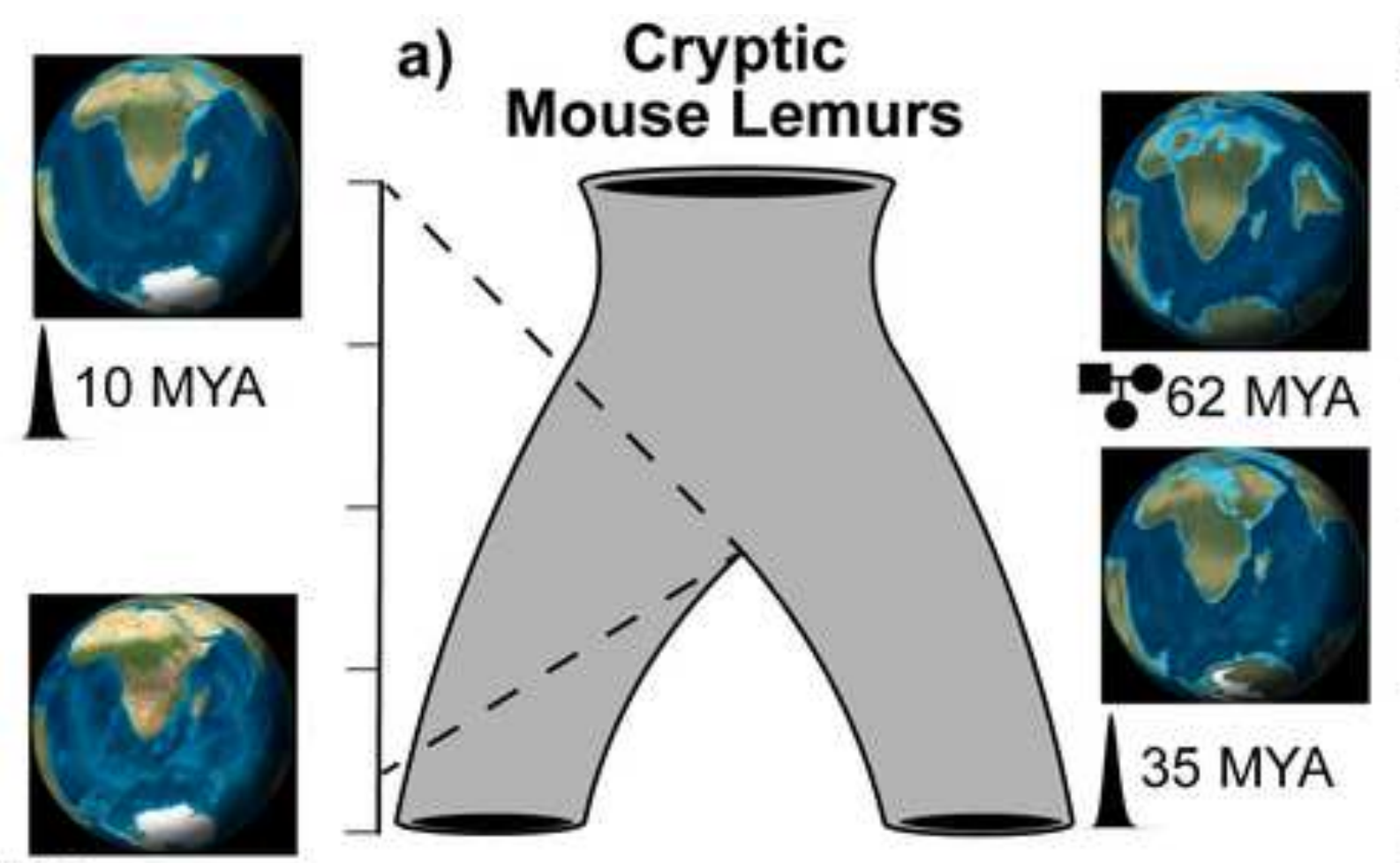

b) Old World Monkeys and Apes

앙.5 MYA
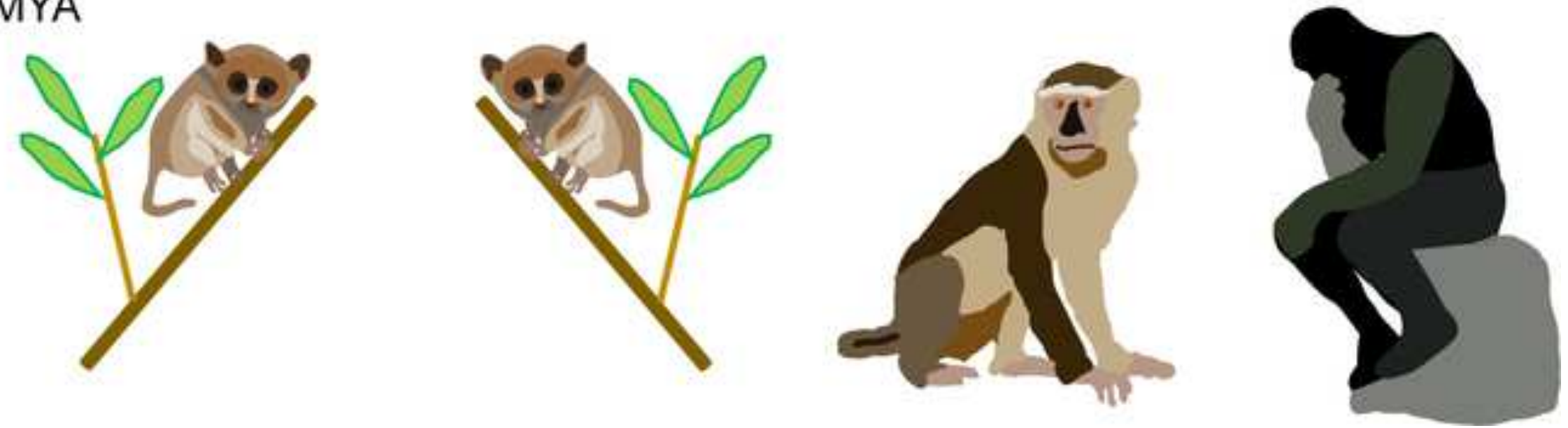
FigureConcatenation with Fossil Calibrations

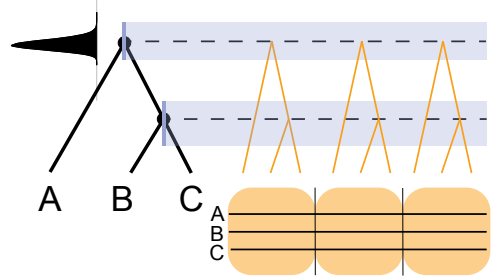

\section{Strengths}

Computationally efficient for large numbers of tips and loci

\section{Weaknesses}

May produce biased estimates when ILS is high or when gene sequence divergence is far from species divergence
MSC with

\section{Fossil Calibrations}

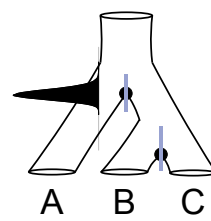

Considers discordance between gene trees and species trees
Increased computational complexity from averaging over gene trees to estimate species tree parameters
Mutation Rate Calibrated MSC
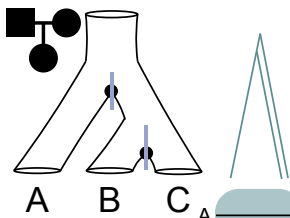

Does not require calibrations on nodes from external information such as fossils

Requires external mutation rate estimates from sequenced pedigrees and potentially not appropriate for distant taxa

\section{Common Programs}

BEAST2 [96]

MCMCTREE [97]

MrBayes [98]

BPP [5,71]

StarBEAST2 [6]
BPP $[5,71]$

StarBEAST2 [6] 